\title{
FANO-MORI CONTRACTIONS OF HIGH LENGTH ON PROJECTIVE VARIETIES WITH TERMINAL SINGULARITIES
}

\author{
MARCO ANDREATTA, LUCA TASIN
}

\begin{abstract}
Let $X$ be a projective variety with $\mathbb{Q}$-factorial terminal singularities and let $L$ be an ample Cartier divisor on $X$.

We prove that if $f$ is a birational contraction associated to an extremal ray $R \subset \overline{N E(X)}$ such that $R .\left(K_{X}+(n-2) L\right)<0$ then $f$ is a weighted blow-up of a smooth point.

We then classify divisorial contractions associated to extremal rays $R$ such that $R .\left(K_{X}+r L\right)<0$, where $r$ is a non-negative integer, and the fibres of $f$ have dimension less or equal to $r+1$.
\end{abstract}

\section{INTRODUCTION}

Let $X$ be a normal projective variety over $\mathbb{C}$ with $\mathbb{Q}$-factorial terminal singularities and let $n=\operatorname{dim} X$.

We consider the Kleiman-Mori cone of $X, \overline{N E(X)}$, which is the closure of the cone generated by effective curves modulo numerical equivalence in $N_{1}(X, \mathbb{R})$. By the famous Cone Theorem of Mori and Kawamata (see Theorem 3.7(1) in [9]), the subcone $\overline{N E(X)} K_{X}<0:=\left\{C \in \overline{N E(X)}: K_{X} . C<0\right\}$ is locally polyhedral. By the Contraction Theorem of Mori, Kawamata and Shokurov, to any extremal ray in $\overline{N E(X)}_{K_{X}<0}$ one can associate a map (contraction) $f: X \rightarrow Z$ with connected fibres onto a normal projective variety $Z$, which contracts all curves in the ray (see Theorem 3.7 in [9]). These contractions are the basic steps of the Minimal Model Program, a program which takes an algebraic variety to a minimal model, i.e a variety on which the canonical class is nef (not negative on any curve).

Choose now a polarization of $X$, that is an ample Cartier divisor $L$ on $X$. Let $r$ be a non-negative rational number. One can consider the subcone $\overline{N E(X)}_{\left(K_{X}+r L\right)<0}=$ $\left\{C \in \overline{N E(X)}:\left(K_{X}+r L\right) . C<0\right\}$. This subcone contains just a finite number of extremal rays (see Theorem 3.7(2) in 9 ). Since $K_{X}+(n+1) L$ is always nef (see for instance 2, Theorem 5.1) we know that if $r \geq n+1$, then the subcone is actually empty, whereas if $r$ approaches 0 , then the subcone fills up all $\overline{N E(X)}_{K_{X}<0}$. Note that the bigger $r$ is, the more negative $K_{X}$ is on the curves of the ray; in these cases the contractions should be simpler.

In a previous paper [2], the first author described all extremal rays contained in the cone $\overline{N E(X)}{ }_{\left(K_{X}+(n-2) L\right)<0}$ : this summarizes and generalizes a series of results, by many authors, of the so called Adjunction Theory (see also [5]).

1991 Mathematics Subject Classification. 14E30, 14J40, 14N30.

We like to thank Edoardo Ballico, Cristiano Bocci, Paolo Cascini, Massimiliano Mella and Roberto Pignatelli for helpful conversations. We thank the referee, whose suggestions remarkably improved the presentation of the paper. 
The first aim of the present paper is to complete the description of the extremal rays $R=\mathbb{R}^{+}[C]$ contained in the cone $\overline{N E(X)}\left(K_{X}+(n-2) L\right)<0$ whose associated contractions are birational. In [2] it was simply proved that the associated contraction contracts a divisor to a smooth point, here we prove that it is always a weighted blow-up, the most straightforward possibility.

Theorem 1.1. Let $X$ be a normal projective variety with $\mathbb{Q}$-factorial terminal singularities and let $L$ be an ample Cartier divisor on $X$. Let $R$ be an extremal ray in $\overline{N E(X)}\left(K_{X}+(n-2) L\right)<0$ and let $f: X \rightarrow Z$ be its associated contraction. Assume that $f$ is birational. Then $f$ is a weighted blow-up of a smooth point with weight $\sigma=(1,1, b, \ldots, b)$, where $b$ is a positive integer (see Definition 3.1).

If $n=3$ the Theorem follows from the results in [2] and the main Theorem in 7]; our proof is however independent of [7].

The Theorem is proved by induction on $n$, starting with case $n=2$, which is Castelnuovo contraction Theorem for $(-1)$-curves on a (smooth) surface. The inductive step is achieved by the usual procedure of Adjunction Theory (the so called Horizontal slicing described in Lemma 2.5), once one has a good divisor in the linear system $|L|$. The existence of such a divisor, i.e. a divisor which has terminal singularities, has been proved in the paper [3].

Secondly, we will include the above Theorem in a more general statement regarding a ray $R=\mathbb{R}^{+}[C]$ contained in the cone $\overline{N E(X)}_{\left(K_{X}+r L\right)<0}$, where $r$ is a non-negative integer, whose associated contraction is divisorial (i.e. it is birational and its exceptional locus is a divisor) with all fibres of dimension less or equal to $r+1$ (in simpler words the fibre dimension of the contraction is not too big compared to the negativity of $R$ ). We prove the following Theorem, which is a generalization of Theorem 4.9 in [8] (see also Theorem 3.2 in [1]).

Theorem 1.2. Let $X$ be a normal projective variety with $\mathbb{Q}$-factorial terminal singularities and let $L$ be an ample Cartier divisor on $X$. Let $R$ be an extremal ray in $\overline{N E(X)}{ }_{\left(K_{X}+r L\right)<0}$ where $r \in \mathbb{N}$ is a non-negative integer and let $f: X \rightarrow Z$ be its associated contraction. Assume that $f$ is divisorial and that all fibres have dimension less or equal to $r+1$. Let $E$ be the exceptional locus of $f$ and set $C:=f(E) \subset Z$.

(1) Then $\operatorname{codim}_{Z} C=r+2$, there is a closed subset $S \subset Z$ of codimension al least 3 such that $Z^{\prime}=Z \backslash S$ and $C^{\prime}=C \backslash S$ are smooth, and $f^{\prime}: X^{\prime}=X \backslash f^{-1}(S) \rightarrow Z^{\prime}$ is a weighted blow-up along $C^{\prime}$ with weight $\sigma=(1,1, b \ldots, b, 0, \ldots, 0)$, where the number of $b$ 's is $r$ (see Definitions 3.1 and 3.9 ).

(2) Let $\mathcal{I}^{\prime}$ be a $\sigma$-weighted ideal sheaf of degree $b$ for $Z^{\prime} \subset X^{\prime}$ (see Definition 3.9) and let $i: Z^{\prime} \rightarrow Z$ be the inclusion; let also $\mathcal{I}:=i_{*}\left(\mathcal{I}^{\prime}\right)$ and $\mathcal{I}^{(m)}$ be the $m$-th symbolic power of $\mathcal{I}$ (see Definition 3.7). Then $X=\operatorname{Proj} \bigoplus_{m \geq 0} \mathcal{I}^{(m)}$.

\section{Contractions}

Our language is compatible with that of [9]. In this section we recall some pertinent definitions and results which are used in the sequel.

A contraction is a surjective morphism, $f: Y \rightarrow T$, between normal varieties and with connected fibres. 
If $\operatorname{dim} Y>\operatorname{dim} T$ the contraction is said to be of fibre type, otherwise it is birational. The set $E=\{y \in Y: f$ is not an isomorphism at $y\}$ is the exceptional locus of $f$. If $f$ is birational and $\operatorname{dim} E=\operatorname{dim} Y-1$, then it is also called a divisorial contraction. For a contraction $f: Y \rightarrow T$, a $\mathbb{Q}$-Cartier divisor $H$ such that $H=\varphi^{*} A$ for some ample $\mathbb{Q}$-Cartier divisor on $T$ is called a supporting divisor for the contraction.

A fundamental result in Mori Theory is the following.

Theorem 2.1 (Contraction Theorem, [9, 3.7(3)]). Let $X$ be a variety with logterminal singularities and let $R \subset \overline{N E(X)}_{K_{X}<0}$ be an extremal ray.

Then there exists a unique projective morphism $\varphi: X \rightarrow W$ onto a normal projective variety $W$ which is characterised by the following properties:

i) For any irreducible curve $C \subset X, \varphi(C)$ is a point if and only if $[C] \in R$,

ii) $\varphi$ has connected fibres.

We call such contraction the Fano-Mori (F-M) contraction associated to $R$; note that $-K_{X}$ is $\varphi$-ample.

In studying the contraction associated to an extremal ray $R$ it makes sense to fix a fibre and understand the contraction locally, i.e. restricting to an affine neighbourhood of the image of the fixed fibre. The complete contraction can then be obtained by gluing different local descriptions.

For this we use the local set-up developed by Andreatta-Wiśniewski, see [3] for the details. Roughly summarizing, let $f: Y \rightarrow T$ be a contraction; fix a fibre $F$ of $f$ and take an open affine $Z \subset T$ such that $f(F) \in Z$ and $\operatorname{dim} f^{-1}(z) \leq \operatorname{dim} F$, for $z \in Z$. If $X=f^{-1} Z$, then $f: X \rightarrow Z$ is called a local contraction around $F$. If there is no need to specify fixed fibres, then we will simply say that $f: X \rightarrow Z$ is a local contraction. Note that in this setting we have $H^{0}\left(X, K_{X}+\tau L\right)=H^{0}\left(X, \mathcal{O}_{X}\right)$ and $Z=\operatorname{Spec}\left(H^{0}\left(X, \mathcal{O}_{X}\right)\right)$. One advantage of the local set-up is that we may always assume that $|L|$ is not empty.

Let $L$ be an ample Cartier divisor and let $R \subset \overline{N E(X)}\left(K_{X}+r L\right)<0$, where $r$ is a non-negative rational number. Let $\varphi: X \rightarrow Z$ be the local contraction around a fibre $F$ associated to $R$.

We can define the nef value of the pair $(X, L)$ as

$$
\tau(X, L):=\inf \left\{t \in \mathbb{R}: K_{X}+t L \text { is } \varphi \text {-nef }\right\} .
$$

By the rationality theorem of Kawamata (Theorem 3.5 in 9 ), $\tau(X, L)$ is a rational non-negative number.

The assumption $R \subset \overline{N E(X)}_{\left(K_{X}+r L\right)<0}$ implies that $\tau>r$.

Moreover note that the $\mathbb{Q}$-Cartier divisor $K_{X}+\tau L$ is a supporting divisor of $\varphi$.

We have a lower bound for the dimension of a fibre of a F-M contraction.

Theorem 2.2 ([1, Theorem 2.1]). Let $\varphi: X \rightarrow Z$ be a local contraction supported by $K_{X}+\tau L$. If $\varphi$ is birational, then $\operatorname{dim} F \geq \tau$.

The following base point free theorem is the main technical tool of the paper.

Theorem 2.3 (3]). Let $\varphi: X \rightarrow Z$ be a local contraction supported by $K_{X}+\tau L$. If $\varphi$ is birational and $\operatorname{dim} F \leq \tau+1$, then $L$ is $\varphi$-base point free. 
By abuse of notation, in the setting of Theorem 2.3. we will sometime say that $L$ is base point free on $X$.

To apply inductive arguments we will need the following two lemmata, which are consequences of Bertini's theorem.

Lemma 2.4 (Vertical slicing, 3, Lemma 2.5]). Let $\varphi: X \rightarrow Z$ be a birational local contraction supported by $K_{X}+\tau L$, where $X$ has terminal singularities and $\tau \geq 0$. Let $X^{\prime}$ be the divisor defined by a general global function $h \in H^{0}\left(X, \mathcal{O}_{X}\right)=$ $H^{0}\left(X, K_{X}+\tau L\right)$. Then $X^{\prime}$ has terminal singularities and $\varphi_{\mid X^{\prime}}: X^{\prime} \rightarrow Z^{\prime}$ is a local contraction supported by $K_{X^{\prime}}+\tau L_{\mid X^{\prime}}$.

Lemma 2.5 (Horizontal slicing, 3, Lemma 2.6]). Let $\varphi: X \rightarrow Z$ be a birational local contraction supported by $K_{X}+\tau L$, where $X$ has terminal singularities and $\tau \geq 1$. Let $X^{\prime} \in|L|$ be a general divisor and let $\varphi^{\prime}=\varphi_{\mid X^{\prime}}: X^{\prime} \rightarrow Z^{\prime}$.

i) Outside of the base locus of $|L|, X^{\prime}$ has terminal singularities.

ii) If $X^{\prime}$ is normal, then $\varphi^{\prime}$ is a local contraction supported by $K_{X^{\prime}}+(\tau-1) L_{\mid X^{\prime}}$.

\section{WeIGHTED BLOW-UPS}

In this section we consider weighted blow-ups along smooth subvarieties; they are a generalization of weighted blow-ups of points as defined, for example, in Section 10 of [9] or in Section 3 of [6].

Let $\sigma=\left(a_{1}, \ldots, a_{k}, 0, \ldots, 0\right) \in \mathbb{N}^{n}$ such that $a_{i}>0$ and $\operatorname{gcd}\left(a_{1}, \ldots, a_{k}\right)=1$. Let $M=\operatorname{lcm}\left(a_{1}, \ldots, a_{k}\right)$. We denote by $\mathbb{P}\left(a_{1}, \ldots, a_{k}\right)$ the weighted projective space with weight $\left(a_{1}, \ldots, a_{k}\right)$, that is the quotient $\mathbb{P}\left(a_{1}, \ldots, a_{k}\right)=\left(\mathbb{A}^{k} \backslash\{0\}\right) / \mathbb{C}^{*}$ where the action on $\mathbb{A}^{k}$ (with coordinates $\left.y_{1}, \ldots, y_{k}\right)$ is given by $\lambda \cdot\left(y_{1}, \ldots, y_{k}\right)=$ $\left(\lambda^{a_{1}} y_{1}, \ldots, \lambda^{a_{k}} y_{k}\right) \quad$ for $\lambda \in \mathbb{C}^{*}$.

Definition 3.1. Let $X=\mathbb{A}^{n}=\operatorname{Spec} \mathbb{C}\left[x_{1}, \ldots, x_{n}\right]$ and $Z=\left\{x_{1}=\ldots=x_{k}=0\right\} \subset$ $X$. Consider the rational map

$$
\varphi: \mathbb{A}^{n} \rightarrow \mathbb{P}\left(a_{1}, \ldots, a_{k}\right)
$$

given by $\left(x_{1}, \ldots, x_{n}\right) \mapsto\left(x_{1}^{a_{1}}: \ldots: x_{k}^{a_{k}}\right)$.

The weighted blow-up of $X$ along $Z$ with weight $\sigma$ is defined as the closure $\bar{X}$ in $\mathbb{A}^{n} \times \mathbb{P}\left(a_{1}, \ldots, a_{k}\right)$ of the graph of $\varphi$, together with the morphism $\pi: \bar{X} \rightarrow X$ given by the projection on the first factor.

The map $\pi$ is birational and contracts an exceptional irreducible divisor $E$ to $Z$. Moreover for any point $z \in Z$ we have $\pi^{-1}(z)=\mathbb{P}\left(a_{1}, \ldots, a_{k}\right)$.

We now describe an affine covering for $\bar{X}$, where each affine set has an orbifold structure; this covering comes out naturally from the Toric construction of the weighted blow-up, as done in [9].

Given integers $b_{1}, \ldots, b_{n}$ we define an action of $\mathbb{Z}_{r}$, the cyclic group of order $r$, on $\mathbb{A}^{n}=\operatorname{Spec} \mathbb{C}\left[x_{1}, \ldots, x_{n}\right]$ by $\tau \cdot x_{i}=\varepsilon^{b_{i}} x_{i}$, where $\tau$ is a generator of $\mathbb{Z}_{r}$ and $\varepsilon$ is a primitive $r^{t h}$-root of unity. The quotient is denoted by $\mathbb{A}^{n} / Z_{r}\left(b_{1}, \ldots, b_{n}\right)$ and it is called a cyclic quotient singularity of type $1 / r\left(b_{1}, \ldots, b_{n}\right)$.

Let $\left(y_{1}: \ldots: y_{k}\right)$ be homogeneous coordinates on $\mathbb{P}\left(a_{1}, \ldots, a_{k}\right)$. For any $i=$ $1, \ldots, k$ consider the open subset $U_{i}=\bar{X} \cap\left\{y_{i} \neq 0\right\} \subset \mathbb{A}^{n} \times \mathbb{P}\left(a_{1}, \ldots, a_{k}\right)$. 
One can see that

$$
\begin{aligned}
U_{i} & \cong \operatorname{Spec} \mathbb{C}\left[\bar{x}_{1}, \ldots, \bar{x}_{n}\right] / \mathbb{Z}_{a_{i}}\left(-a_{1}, \ldots, \stackrel{i \text {-th }}{1}, \ldots,-a_{k}, 0, \ldots, 0\right) \\
& \cong\left(\operatorname{Spec} \mathbb{C}\left[\bar{x}_{1}, \ldots, \bar{x}_{k}\right] / \mathbb{Z}_{a_{i}}\left(-a_{1}, \ldots, \stackrel{i \text {-th }}{1}, \ldots,-a_{k}\right)\right) \times \mathbb{A}^{n-k} .
\end{aligned}
$$

and

$$
\pi_{\mid U_{i}}: U_{i} \ni\left(\bar{x}_{1}, \ldots, \bar{x}_{n}\right) \mapsto\left(\bar{x}_{1} \bar{x}_{i}^{a_{1}}, \ldots, \bar{x}_{i}^{a_{i}}, \ldots, \bar{x}_{k} \bar{x}_{i}^{a_{k}}, \bar{x}_{k+1}, \ldots, \bar{x}_{n}\right) \in X .
$$

In the affine set $U_{i}, E$ is defined by $\left\{\bar{x}_{i}=0\right\} / \mathbb{Z}_{a_{i}}\left(-a_{1}, \ldots, 1, \ldots,-a_{k}, 0, \ldots, 0\right)$; hence $M E$ is a Cartier divisor on $\bar{X}$.

We define the function

$$
\sigma \text {-wt }: \mathbb{C}\left[x_{1}, \ldots, x_{n}\right] \rightarrow \mathbb{N}
$$

as it follows. For a monomial $T=x_{1}^{s_{1}} \ldots x_{n}^{s_{n}}$ we set $\sigma$-wt $(T):=\sum_{i=1}^{k} s_{i} a_{i}$. For a polynomial $f=\sum_{I} \alpha_{I} T_{I}$, where $\alpha_{I} \in \mathbb{C}$ and $T_{I}$ are monomials, we set

$$
\sigma \text {-wt }:=\min \left\{\sigma-\mathrm{wt}\left(T_{I}\right): \alpha_{I} \neq 0\right\} .
$$

Definition 3.2. Let $\sigma=\left(a_{1}, \ldots, a_{k}, 0, \ldots, 0\right) \in \mathbb{N}^{n}$ such that $a_{i}>0$ and $\operatorname{gcd}\left(a_{1}, \ldots, a_{k}\right)=$ 1. For any $d \in \mathbb{N}$ we define the $\sigma$-weighted ideal of degree $d$ as

$$
I_{\sigma, d}=\left\{g \in \mathbb{C}\left[x_{1}, \ldots, x_{n}\right]: \sigma-w t(g) \geq d\right\}=\left(x_{1}^{s_{1}} \cdots x_{n}^{s_{n}}: \sum_{j=1}^{k} s_{j} a_{j} \geq d\right) .
$$

Lemma 3.3. Let $\pi: \bar{X} \rightarrow X$ be the weighted blow-up of $X=\mathbb{A}^{n}$ along $Z=\left\{x_{1}=\right.$ $\left.\ldots=x_{k}=0\right\}$ with weight $\sigma=\left(a_{1}, \ldots, a_{k}, 0, \ldots, 0\right)$. Then

$$
\pi_{*} \mathcal{O}_{X}(-d E)=I_{\sigma, d}
$$

Therefore

$$
\bar{X}=\operatorname{Proj} \bigoplus_{d \geq 0} I_{\sigma, d}
$$

Proof. Let $\left\{U_{i}\right\}$ be the standard affine covering of $\bar{X}$.

As the exceptional divisor $E$ is effective, we have that $J:=\pi_{*} \mathcal{O}_{X}(-d E) \subset$ $\mathbb{C}\left[x_{1}, \ldots, x_{n}\right]$ is an ideal. A polynomial $g\left(x_{1}, \ldots, x_{n}\right)$ is an element of $J$ if and only if for any $1 \leq i \leq k$ we have that $g\left(\bar{x}_{1}, \ldots, \bar{x}_{n}\right) \in \Gamma\left(U_{i}, \mathcal{O}_{X}(-d E)\right)$. Since $E$ is defined by $\left\{\bar{x}_{i}=0\right\} / \mathbb{Z}_{a_{i}}$ on the affine subset $U_{i}$, we have that $\bar{x}_{i}^{d}$ divides $g\left(\bar{x}_{1}, \ldots, \bar{x}_{n}\right)$ if and only if $\sigma$-wt $(g) \geq d$, and the lemma follows.

In the case of the blow-up with weight $(1,1, b, \ldots, b, 0 \ldots, 0)$ we can say something more.

Proposition 3.4. Let $\pi: \bar{X} \rightarrow X$ be the weighted blow-up of $X=\mathbb{A}^{n}$ along $Z=\left\{x_{1}=\ldots=x_{k}=0\right\}$ with weight $\sigma=(1,1, b, \ldots, b, 0, \ldots, 0)$ (where the number of $b$ 's is $k-2)$. Then

$$
\pi_{*} \mathcal{O}_{X}(-d b E)=\pi_{*} \mathcal{O}_{X}(-b E)^{d}
$$

and

$$
\bar{X}=\operatorname{Proj} \bigoplus_{d \geq 0} I_{\sigma, b}^{d}
$$


Proof. It suffices to prove that for every integer $d \geq 1$ the natural map

$$
\pi_{*} \mathcal{O}_{X}(-(d-1) b E) \bigotimes \pi_{*} \mathcal{O}_{X}(-b E) \rightarrow \pi_{*} \mathcal{O}_{X}(-d b E)
$$

is surjective. For $d=1$ there is nothing to prove, so we assume $d \geq 2$.

Let $g=x_{1}^{s_{1}} \cdots x_{n}^{s_{n}} \in \pi_{*} \mathcal{O}_{X}(-d b E)=\left(x_{1}^{s_{1}} \cdots x_{n}^{s_{n}}: s_{1}+s_{2}+\sum_{i=3}^{k} s_{i} b \geq d b\right)$. We claim that there exists $h=x_{1}^{t_{1}} \cdots x_{n}^{t_{n}} \in \pi_{*} \mathcal{O}_{X}(-b E)$ such that $t_{1}+t_{2}+\sum_{i=3}^{k} t_{i} b=b$ and $t_{i} \leq s_{i}$ for all $1 \leq i \leq n$.

In fact, if there is $j \in\{3, \ldots, k\}$ such that $s_{j} \neq 0$, then just set $h=x_{j}$. If $s_{j}=0$ for $j=3, \ldots, k$, then either $s_{1} \geq b$ or $s_{2} \geq b$ and the claim follows setting $h=x_{1}^{b}$ or $h=x_{2}^{b}$.

Let $k=g \cdot h^{-1}$, then $k \in \pi_{*} \mathcal{O}_{X}(-(d-1) b E)$ and $g=k \cdot h$.

The second equality is a consequence of the first equality and Lemma 3.3

We remark that the previous Proposition does not hold for any weight $\sigma$ as the following example shows. Nevertheless, since the algebra

$$
\bigoplus_{d \geq 0} I_{\sigma, b}^{d}
$$

is finitely generated, there is always a positive integer $L$ such that

$$
I_{\sigma, L}^{d}=I_{\sigma, d L} .
$$

for any $d \in \mathbb{N}$.

Example 3.5. Let $Z=\left\{x_{1}=x_{2}=x_{3}=0\right\} \subset X=\mathbb{A}^{n}$ and $\sigma=(10,14,35,0, \ldots, 0)$. Let $\pi: \bar{X} \rightarrow X$ be the weighted blow-up of $X$ along $Z$ with weight $\sigma$. Consider

$$
g=x_{1}^{5} x_{2}^{4} x_{3} \in \pi_{*} \mathcal{O}_{X}(-2 M E),
$$

where $M=\operatorname{lcm}(2,5,7)=70$; note that $\sigma$-wt $(g)=141$. It is easy to check that there is no triple $\left(t_{1}, t_{2}, t_{3}\right) \in \mathbb{N}^{3}$ such that $10 t_{1}+14 t_{2}+35 t_{1}=70$ and $t_{1} \leq 5, t_{2} \leq 4$, $t_{3} \leq 1$ and hence

$$
\pi_{*} \mathcal{O}_{X}(-140 E) \neq \pi_{*} \mathcal{O}_{X}(-70 E)^{2} .
$$

Lemma 3.6. Let $\pi: \bar{X} \rightarrow X$ be the weighted blow-up of $X=\mathbb{A}^{n}$ along $Z=\left\{x_{1}=\right.$ $\left.\ldots=x_{k}=0\right\}$ with weight $\sigma=(1,1, b, \ldots, b, 0, \ldots, 0)$ (where the number of $b$ 's is $k-2)$. Then $\bar{X}$ has terminal singularities.

Proof. By the above description of the open subsets $U_{i} \subset \bar{X}$ we must show that a cyclic quotient singularity of type $\frac{1}{b}(b-1, b-1,1,0, \ldots, 0)$ is terminal. This follows immediately by [14, Thm. 4.11].

We now define the symbolic powers of an ideal and check that $\sigma$-weighted ideals behave well with respect to symbolic powers.

Definition 3.7. Given an ideal $I \subset R$ in a Noetherian ring $R$ and $t \in \mathbb{N}$, the $t$-th symbolic power $I^{(t)}$ of $I$ is defined as the restriction of $I^{t} R_{S}$ to $R$, where $S$ is the complement of the union of the minimal associated primes of $I$ and $R_{S}$ is the localization of $R$ at the multiplicative system $S$.

If $I$ is a prime ideal, then the definition of symbolic power is for instance given in [10, Definition 9.3.4] or in [4, Exercise 4.13]. Note that, by definition, $I^{t} \subset I^{(t)}$; in general the inclusion might be strict. 
Lemma 3.8. Let $\sigma=\left(a_{1}, \ldots, a_{k}, 0 \ldots, 0\right) \in \mathbb{N}^{n}$ such that $a_{i}>0$ and $\operatorname{gcd}\left(a_{1}, \ldots, a_{k}\right)=$ 1 , and let $L$ be a positive integer such that

$$
I_{\sigma, L}^{d}=I_{\sigma, d L} .
$$

for any $d \in \mathbb{N}$, where $I_{\sigma, d}$ is the $\sigma$-weighted of degree $d$. Set $I=I_{\sigma, L}$.

Then for any $t \in \mathbb{N}$ we have $I^{t}=I^{(t)}$.

Proof. We will use the fact that if $f, g \in R$ then $\sigma$-wt $(f g)=\sigma$-wt $(f)+\sigma$-wt $(g)$.

We first show that $I$ is primary: if $f g \in I$ then $\sigma$-wt $(f) \geq 1$ or $\sigma$-wt $(g) \geq 1$ and hence $f^{m} \in I$ or $g^{m} \in I$ for $m$ big enough. Then the only prime associate to $I$ is its radical ideal $r(I)$, which is $r(I)=\left(x_{1}, \ldots, x_{k}\right)$ since there is always a power of $x_{i}$ in $I$ for $1 \leq i \leq k$.

Let now $S=R \backslash r(I)$. By Proposition 3.11 in [4] we have $I^{(t)}=\bigcup_{s \in S}\left(I^{t}: s\right)$. Using the fact that $\sigma$-wt $(s)=0$, we have that for any $s \in S$

$$
\left(I^{t}: s\right)=\{g \in R: \sigma-w t(g s) \geq t L\}=\{g \in R: \sigma-w t(g) \geq t L\}=I^{t} .
$$

The definition of weighted blow-up in 3.1 depends on the local coordinates chosen. To construct a global weighted blow-up along a subvariety $Z$ of a complete variety $X$ one needs to patch together weighted blow-ups defined on a covering of $X$, in such a way that the local coordinates preserve the weight. We propose the following.

Definition 3.9. Let $X$ be a smooth variety and $Z$ a smooth subvariety of codimension $k$ and let $\sigma=\left(a_{1}, \ldots, a_{k}, 0 \ldots, 0\right) \in \mathbb{N}^{n}$ such that $a_{i}>0$ and $\operatorname{gcd}\left(a_{1}, \ldots, a_{k}\right)=$ 1. Let $\mathcal{I}_{\sigma, d}$ be ideal sheaves on $X$ such that there is a covering $\left\{U_{i} \cong \mathbb{C}^{n}\right\}_{i \in I}$ on $X$ so that for any $i \in I$ there are local coordinates $x_{1}, \ldots, x_{n}$ on $U_{i}$ for which $Z \cap U_{i}=\left\{x_{1}=\ldots=x_{k}=0\right\}$ and $\Gamma\left(U, \mathcal{I}_{\sigma, d}\right)=\left\{g \in \mathbb{C}\left[x_{1}, \ldots, x_{n}\right]: \sigma-w t(g) \geq d\right\}$. $A$ weighted blow-up of $X$ along $Z$ with weight $\sigma$ is the projectivization

$$
\pi: \bar{X}=\operatorname{Proj} \bigoplus_{d \geq 0} \mathcal{I}_{\sigma, d} \rightarrow X
$$

We call $\mathcal{I}_{\sigma, d}$ a $\sigma$-weighted ideal sheaf of degree $d$ for $Z$ in $X$.

Remark 3.10. Let $Z \subset X$ be a smooth subvariety of codimension $k$; let also $\sigma=\left(a_{1}, \ldots, a_{k}, 0, \ldots, 0\right)$ be a weight. The question about the existence of a $\sigma$ weighted ideal for $Z$ in $X$, and therefore of a weighted blow-up of $X$ along $Z$ with weight $\sigma$, is not clear. In general it seems a difficult problem to find sufficient conditions for a positive answer.

However, if $Z \subset \mathbb{P}^{n}$ is a complete intersection, then for any weight $\sigma=\left(a_{1}, \ldots, a_{k}, 0, \ldots, 0\right)$ there exists a weighted blow-up along $Z$ with weight $\sigma$. In fact, let $F_{1}, \ldots, F_{k}$ be a regular sequence of homogeneous polynomials generating the ideal $I_{Z} \subset R=$ $\mathbb{C}\left[x_{0}, \ldots, x_{n}\right]$ of $Z$. Then we define a function, $\sigma$-wt, on $\mathbb{C}\left[x_{0}, \ldots, x_{n}\right]$ as follows. If $g \notin I_{Z}$ then just set $\sigma$-wt $(g)=0$. If $g \in I_{Z}$, then write $g=\sum_{\beta \in \mathbb{N}^{k}} h_{\beta} F_{1}^{\beta_{1}} \cdots F_{k}^{\beta_{k}}$, where $\beta=\left(\beta_{1}, \ldots, \beta_{k}\right) \in \mathbb{N}^{k}$ and $h_{\beta} \in R \backslash I_{Z}$, and set

$$
\sigma-w t(g):=\min _{\beta}\left\{\sum_{i=1}^{k} a_{i} \beta_{i}: h_{\beta} \neq 0\right\} .
$$


Note that the function $\sigma$-wt is a well defined because $F_{1}, \ldots, F_{k}$ is a regular sequence.

For any $d \in \mathbb{N}$, we define the $\sigma$-weighted ideal sheaf $\mathcal{I}_{\sigma, d} \subset \mathcal{O}_{X}$ setting, for any open subset $U \subset \mathbb{P}^{n}$,

$\Gamma\left(U, \mathcal{I}_{\sigma, d}\right)=\left\{\frac{f}{g}: f, g \in \mathbb{C}\left[x_{0}, \ldots, x_{n}\right]\right.$ are homogeneous, $g_{\mid U} \not \equiv 0$ and $\left.\sigma-w t(f)-\sigma-w t(g) \geq d\right\}$.

\section{Proofs}

Proof of Theorem 1.1. Let $F_{1}$ be a non trivial fibre of $f$. We pass to a local set-up, i.e. we assume that $f: X \rightarrow Z$ is a local F-M contraction around $F_{1}$ supported by $K_{X}+\tau L$, where $\tau$ is the nef value of the pair $(X, L)$, a positive rational number greater than $n-2$. Let $F$ be a component of $F_{1}$. By Theorem 2.2 $\operatorname{dim} F \geq \tau>n-2$; this means that $\operatorname{dim} F=n-1$ and hence the exceptional locus of $f$ has codimension 1. Since the exceptional locus of a divisorial contraction is irreducible we conclude that $F$ is the exceptional divisor, and $f$ is the contraction of $F$ to a point $p \in Z$.

Since $f$ is a $K_{X}$-negative contraction, $Z$ is terminal and $\mathbb{Q}$-factorial (see 9 , Corollary 3.43]). Proposition 3.6 of [2] says that $p$ is smooth. For the reader's convenience we repeat that proof. More precisely, by induction on $n \geq 2$, we prove that $p$ is smooth in $Z$ and that

$$
K_{X}=f^{*} K_{Z}+((n-2) b+1) F \text { and } \tau=n-2+\frac{1}{b}
$$

for a positive integer $b$ such that $f^{*} f_{*} L=L+b F$.

If $n=2, X$ is smooth and in this case we can apply Castelnuovo's theorem, which says that $f$ is the contraction of a $(-1)$-curve $F$ to a smooth surface $Z$, therefore $K_{X}=f^{*} K_{Z}+F$. Note that $L_{1}:=f_{*} L$ is a Cartier divisor on $Z$ and there is a positive integer $b$ such that $L=f^{*} L_{1}-b F$. From $0=\left(K_{X}+\tau L\right) . F=$ $\left(K_{X}-\tau b F\right) \cdot F=-1+\tau b$, we get $\tau=1 / b$.

Let $n \geq 3$ and pick a general member $X^{\prime} \in|L|$ : by Theorem 2.3 and Bertini's theorem it has terminal $\mathbb{Q}$-factorial singularities. Consider the restricted morphism $f^{\prime}:=f_{\mid X^{\prime}}: X^{\prime} \rightarrow Z^{\prime}$; by Lemma 2.5 it is a divisorial contraction supported by $K_{X^{\prime}}+(\tau-1) L_{\mid X^{\prime}}$. By inductive assumption, $p \in Z^{\prime}$ is smooth; by [11, Lemma 1.7] we conclude that $p \in Z$ is smooth, $L_{1}:=f_{*} L$ is Cartier and $L=f^{*} L_{1}-b F$ for a positive integer $b$. Denoting by $F^{\prime}$ the exceptional divisor of $f^{\prime}$, by induction we have

$$
K_{X^{\prime}}=f^{*} K_{Z^{\prime}}+((n-3) b+1) F^{\prime} \text { and } \tau-1=n-3+\frac{1}{b},
$$

from which (4.0.1) follows.

Let $X^{\prime} \in|L|$ be again a general element and $f^{\prime}:=f_{\mid X^{\prime}}: X^{\prime} \rightarrow Z^{\prime}$ be the restricted morphism. Since $Z$ and $Z^{\prime}=f_{*} X^{\prime}$ are smooth at $p$ and $f$ is local, we may assume that $Z=\mathbb{A}^{n}=\operatorname{Spec} \mathbb{C}\left[x_{1}, \ldots, x_{n}\right]$, where $x_{1}, \ldots, x_{n}$ are local coordinates for $p$, and that $f_{*} X^{\prime}=\left\{x_{n}=0\right\}$.

Note that $\mathcal{O}_{X}(-b F)$ is $f$-ample and that the map $f$ is proper; so we have that

$$
X=\operatorname{Proj}\left(\oplus_{d \geq 0} f_{*} \mathcal{O}_{X}(-d b F)\right) .
$$

By Lemma $3.3 X$ will be the weighted blow-up we are looking for if

$$
f_{*} \mathcal{O}_{X}(-d b F)=I_{\sigma, d}=\left(x_{1}^{s_{1}} \cdots x_{n}^{s_{n}}: s_{1}+s_{2}+\sum_{j=3}^{n} b s_{j} \geq d b\right) .
$$


The proof of this is by double induction on $n$ and $d$, starting with $n=2$ and $d=0$.

Consider the exact sequence

$$
0 \rightarrow \mathcal{O}_{X}(-L-d b F) \rightarrow \mathcal{O}_{X}(-d b F) \rightarrow \mathcal{O}_{X^{\prime}}(-d b F) \rightarrow 0 .
$$

Note that

$$
-L-d b F \sim_{f}-(d-1) b F \sim_{f} K_{X}+\left(n-3+d+\frac{1}{b}\right) L .
$$

Hence, pushing down to $Z$ the above exact sequence and applying the relative Kawamata-Viehweg Vanishing, we have

$$
0 \rightarrow f_{*} \mathcal{O}_{X}(-(d-1) b F) \stackrel{\cdot x_{n}}{\rightarrow} f_{*} \mathcal{O}_{X}(-d b F) \rightarrow f_{*} \mathcal{O}_{X^{\prime}}(-d b F) \rightarrow 0 .
$$

By induction on $n$, we can assume that

$$
f_{*} \mathcal{O}_{X^{\prime}}(-d b F)=\left(x_{1}^{s_{1}} \cdots x_{n-1}^{s_{n-1}}: s_{1}+s_{2}+\sum_{j=3}^{n-1} b s_{j} \geq d b\right)
$$

where $s_{j} \in \mathbb{N}$. The case $n=2$ follows from Castelnuovo's theorem. By induction on $d$, we can also assume that

$$
f_{*} \mathcal{O}_{X}(-(d-1) b F)=\left(x_{1}^{s_{1}} \cdots x_{n}^{s_{n}}: s_{1}+s_{2}+\sum_{j=3}^{n} b s_{j} \geq(d-1) b\right),
$$

the case $d=0$ being trivial.

Let $g=x_{1}^{s_{1}} \cdots x_{n}^{s_{n}} \in f_{*} \mathcal{O}_{X}(-d b F)$ be a monomial.

If $s_{n} \geq 1$ then $g$, looking at the sequence (4.0.2), comes from $f_{*} \mathcal{O}_{X}(-(d-1) b F)$ by the multiplication by $x_{n}$; therefore

$$
s_{1}+s_{2}+\sum_{j=3}^{n-1} s_{j} b+s_{n} b \geq(d-1) b+s_{n} b \geq d b .
$$

If $s_{n}=0$, then $g \in f_{*} \mathcal{O}_{X^{\prime}}(-d b F)$ and so

$$
s_{1}+s_{2}+\sum_{j=3}^{n} s_{j} b=s_{1}+s_{2}+\sum_{j=3}^{n-1} s_{j} b \geq d b .
$$

The non-monomial case follows immediately.

To prove Theorem 1.2 we need some preliminary lemmata.

The following is a local version of Theorem 1.2 around a general fibre.

Lemma 4.1. Let $f: X \rightarrow Z$ be a local contraction supported by $K_{X}+\tau L$, where $X$ is terminal $\mathbb{Q}$-factorial and $L$ is an $f$-ample Cartier divisor. Assume that $f$ is divisorial and let $E$ be the exceptional divisor. Set $C=F(E) \subset Z$. Assume also that there exists a positive integer $r$ such that $\tau>r$ and that any fibre of $f$ has dimension less or equal to $r+1$. Let $F$ be a general nontrivial fibre. Then $f(F)$ is a smooth point and, possibly shrinking $Z$ to a smaller affine neighbourhood of $f(F)$ and choosing appropriate coordinates, we have that $C=\left\{x_{1}=\ldots=x_{r+2}=0\right\} \subset$ $\mathbb{C}^{n}=Z$ and $f$ is a weighted blow-up along $C$ with weight $\sigma=(1,1, b, \ldots, b, 0 \ldots, 0)$, where $b$ is a positive integer and the number of $b$ 's is $r$. 
Proof. By the assumptions and by Theorem 2.2, we gain that $\operatorname{dim} F=r+1$; therefore $\operatorname{dim} C=n-r-2$.

First we prove that $p=f(F)$ is a smooth point of $Z$. Take $n-r-2$ general functions $h_{j} \in H^{0}\left(X, \mathcal{O}_{X}\right), j=1, \ldots, n-r-2$ and let $X_{j} \subset X$ be the divisor defined by $h_{j}$. By Lemma 2.4 setting $X "=\bigcap_{j=1}^{n-r+2} X_{j}$, we have that $f ":=f_{\mid X}$ ": $X " \rightarrow Z$ " is a local contraction supported by $K_{X}$ " $+\tau L_{X}$ ", it is birational, it contracts a divisor $F$ to the point $p=C \cap Z$ " and $\tau>r=\operatorname{dim} X "-2$. Note that $p$ is general in $C$ and hence $F$ is a general nontrivial fibre of $f$. The contraction $f ": X " \rightarrow Z$ " satisfies the assumption of Theorem 1.1 and hence we have that $f$ " is a weighted blow-up of a smooth point with weight $(1,1, b, \ldots, b)$, where $b$ is a positive integer.

Therefore we may assume that $Z "=f "(X ")$ is smooth at $p$. Since $Z$ " is an intersection of Cartier divisors in $Z$, we conclude that $Z$ is smooth at $p$.

The proof now is by induction on the dimension of $F$, i.e. on $\operatorname{dim} F=r+1$; for this we apply Lemma 2.5.

Assume $\operatorname{dim} F=1$. Since $X$ has terminal singularities, which are in codimension $3, F$ is contained in the smooth locus of $X$, and hence, in the local set-up, we may assume that $X$ is smooth. Therefore $X$ is a smooth blow-up (see for instance Corollary 4.11 in [3]): i.e. $f$ is the blow-up of $C=\left\{x_{1}=x_{2}=0\right\} \subset Z$ with weights $(1,1,0 \ldots, 0)$; in particular we have that

$$
K_{X}=f^{*} K_{Z}+E .
$$

If $\operatorname{dim} F=r+1 \geq 2$, let $X^{\prime}$ be a general element in $|L|$. By Theorem 2.3 and Bertini's theorem, $X^{\prime}$ has terminal $\mathbb{Q}$-factorial singularities. Consider the contraction $f^{\prime}:=f_{\mid X^{\prime}}: X^{\prime} \rightarrow Z^{\prime}$; by Lemma 2.5, it is a divisorial contraction supported by $K_{X^{\prime}}+(\tau-1) L_{\mid X^{\prime}}$ with fibres of dimension equal to $r$. We have already proved that we may assume $Z=\mathbb{C}^{n}$ and that $Z^{\prime}$ is smooth, therefore, by induction, we may assume $C=\left\{x_{1}=\ldots=x_{r+2}=0\right\} \subset Z^{\prime}=\left\{x_{r+2}=0\right\} \subset Z^{\prime}$ and that $f^{\prime}$ is the smooth blow-up along $C$.

Let $L_{1}$ be the Cartier divisor $f_{*} L$; we have $L=f^{*} L_{1}-b E$ for a positive integer $b$ and $b E$ is a Cartier divisor.

Reasoning as in the proof of Theorem 1.1, by horizontal slicing and by induction on $r$, we get the formulas

$$
K_{X}=f^{*} K_{Z}+(r b+1) E \quad \text { and } \quad \tau=r+\frac{1}{b} .
$$

Since $\mathcal{O}_{X}(-b E)$ is $f$-ample we have

$$
X=\operatorname{Proj} \oplus_{d \geq 0} f_{*} \mathcal{O}_{X}(-d b E) .
$$

By Lemma 3.3 we have to show that

$$
f_{*} \mathcal{O}_{X}(-d b E)=I_{\sigma, d}=\left(x_{1}^{s_{1}} \cdots x_{n}^{s_{n}}: s_{1}+s_{2}+\sum_{j=3}^{r+2} s_{j} b \geq d b\right) .
$$

The proof is by double induction on $r \geq 0$ and $d \geq 0$, and it is similar to the proof of Theorem 1.1

Consider the exact sequence

$$
0 \rightarrow \mathcal{O}_{X}(-L-d b E) \rightarrow \mathcal{O}_{X}(-d b E) \rightarrow \mathcal{O}_{X^{\prime}}(-d b E) \rightarrow 0 .
$$


Note that

$$
-L-d b E \sim_{f} K_{X}+\left(r+(d-1)+\frac{1}{b}\right) L
$$

and hence, by the Relative Kawamata-Viehweg Vanishing, we have

$$
0 \rightarrow f_{*} \mathcal{O}_{X}(-(d-1) b E) \stackrel{\cdot x_{r+2}}{\rightarrow} f_{*} \mathcal{O}_{X}(-d b E) \rightarrow f_{*} \mathcal{O}_{X^{\prime}}(-d b E) \rightarrow 0 .
$$

By induction on $r$ we can assume that

$$
f_{*} \mathcal{O}_{X^{\prime}}(-d b E)=\left(x_{1}^{s_{1}} \cdots x_{r+1}^{s_{r+1}} x_{r+3}^{s_{r+3}} \cdots x_{n}^{s_{n}}: s_{1}+s_{2}+\sum_{j=3}^{r+1} s_{j} b \geq d b\right)
$$

where $s_{j} \in \mathbb{N}$. We have already treated above the case $r=0$. By induction on $d \geq 0$, we can assume that

$$
f_{*} \mathcal{O}_{X}(-(d-1) b E)=\left(x_{1}^{s_{1}} \cdots x_{n}^{s_{n}}: s_{1}+s_{2}+\sum_{j=3}^{r+2} s_{j} b \geq(d-1) b\right),
$$

the case $d=0$ being trivial.

Let $g=x_{1}^{s_{1}} \cdots x_{n}^{s_{n}} \in f_{*} \mathcal{O}_{X}(-d b E)$.

If $s_{r+2} \geq 1$ then, looking at the sequence (4.0.3), $g$ comes from $f_{*} \mathcal{O}_{X}(-(d-1) d E)$ by the multiplication by $x_{r+2}$ and so

$$
s_{1}+s_{2}+\sum_{j=3}^{r+1} s_{j} b+s_{r+2} b \geq(d-1) b+s_{r+2} \geq d b .
$$

Otherwise $g \in f_{*} \mathcal{O}_{X^{\prime}}(-b d E)$ and it satisfies

$$
s_{1}+s_{2}+\sum_{j=3}^{r+1} s_{j} b=s_{1}+s_{2}+\sum_{j=3}^{r} s_{j} b \geq d b .
$$

Proof of Theorem 1.2. First notice that, as in the first line of the proof of 1.1 $\operatorname{dim} C=n-r-2$.

We proceed as in [8, Theorem 4.9].

By Lemma 4.1, $C \cap \operatorname{Sing}(Z) \subsetneq C$; moreover since $Z$ has terminal singularities, $\operatorname{Sing}(Z)$ has codimension at least three. Therefore we can find a codimension three closed subset $S \subset Z$ such that $Z^{\prime}=Z \backslash S$ is smooth and $f^{-1}(S)$ has codimension at least two in $X$. Moreover, by Lemma 4.1 we may assume that $X^{\prime}=X \backslash f^{-1}(S)$ is a weighted blow-up of $Z^{\prime}$ along $C^{\prime}=C \backslash S$ with weight $\sigma=(1,1, b, \ldots, b, 0, \ldots, 0) \in$ $\mathbb{N}^{n}$. This proves (i).

To prove (ii), since $X=\operatorname{Proj} \bigoplus_{m \geq 0} f_{*} \mathcal{O}_{X}(-m b E)$, we need to show that

$$
f_{*} \mathcal{O}_{X}(-m b E)=\mathcal{I}^{(m)} .
$$

Note that by Proposition 3.4 we have

$$
f_{*}\left(\mathcal{O}_{X}(-m b E)\right)_{\mid Z^{\prime}}=\left(\mathcal{I}_{\mid Z^{\prime}}\right)^{m} .
$$

By definition of symbolic power, and using the fact proved in Lemma 3.8 that $\left(\mathcal{I}_{\mid Z^{\prime}}\right)^{m}=\left(\mathcal{I}_{\mid Z^{\prime}}\right)^{(m)}=\left(\mathcal{I}^{(m)}\right)_{\mid Z^{\prime}}$, we obtain 
Therefore 4.0.4 follows by

$$
i_{*}\left(\left(\mathcal{I}^{(m)}\right)_{\mid Z^{\prime}}\right)=\mathcal{I}^{(m)} .
$$

$$
i_{*}\left(f_{*}\left(\mathcal{O}_{X}(-m b E)\right)_{\mid Z^{\prime}}\right)=f_{*}\left(\mathcal{O}_{X}(-m b E)\right),
$$

which is a consequence of the following general fact.

Lemma 4.2. Let $f: U \rightarrow V$ be a proper morphism. Let $S \subset V$ be a closed subset such that the codimension of $f^{-1}(S)$ in $U$ is at least two. Let $\mathcal{F}$ be a sheaf that satisfies Serre's condition $S_{2}$ (e.g. $U$ is normal and $\mathcal{F}$ is reflexive). Then $f_{*} \mathcal{F}=i_{*}\left(f_{*} \mathcal{F}_{\mid V \backslash S}\right)$, where $i: V \backslash S \rightarrow V$ is the injection.

\section{REFERENCES}

[1] BibliographyM. Andreatta. Some remarks on the study of good contractions, Manuscripta Math., volume 87, 1995, 359-367.

[2] BibliographyM. Andreatta. Minimal Model Program with scaling and adjunction theory. Internat. J. M. Vol. 24 (2013), no. 02.

[3] BibliographyM. Andreatta and J. A. Wiśniewski. A note on nonvanishing and applications, Duke Math. J., volume 72 n. 3, 1993, 739-755.

[4] BibliographyM. F. Atiyah and I. G. Macdonald. Introduction to commutative algebra, Addison-Wesley Publishing Co., Reading, Mass.-London-Don Mills, Ont. 1969 ix+128 pp.

[5] BibliographyM. Beltrametti and A.J. Sommese. The Adjunction Theory of Complex Projective Varieties, volume 16 of Expositions in Mathematics, De Gruyter, Berlin-New York, 1995.

[6] BibliographyT. Hayakawa. Blowing ups of 3-dimensional terminal singularities, Publ. Res. Inst. Math. Sci. 35 (1999), 515-570.

[7] BibliographyM. Kawakita. Divisorial contractions in dimension three which contract divisors to smooth points, Invent. Math. vol. 145, no. 1, 2001, 105-119.

[8] BibliographyJ. Kollár and S. Mori. Classification of three-dimensional flips. J. Amer. Math. Soc. 5 (1992), no. 3, 533-703. 14E30 (14B07 14E05 14E35 14J30)

[9] BibliographyJ. Kollár and S. Mori. Birational geometry of algebraic varieties, volume 134 of Cambridge Tracts in Mathematics. Cambridge University Press, Cambridge, 1998. With the collaboration of C. H. Clemens and A. Corti, Translated from the 1998 Japanese original.

[10] BibliographyR. Lazarsfeld. Positivity in Algebraic Geometry II. Ergebnisse der Mathematik und ihrer Grenzgebiete, vol. 49, Springer-Verlag, Berlin, 2004.

[11] BibliographyM. Mella. Adjunction theory on terminal varieties. Complex analysis and geometry (Trento, 1995), 153-164, Pitman Res. Notes Math. Ser., 366, Longman, Harlow, 1997.

[12] BibliographyS. Mori. Threefolds whose canonical bundles are not numerically effective, $A n$ nals of Math, vol. 116, 1982, 133-176.

[13] BibliographyS. Mori. Flip theorem and the existence of minimal models for 3-folds, Jour. AMS, vol. 1, 1998, 117-253.

[14] BibliographyM. Reid. Young person's guide to canonical singularities. Algebraic geometry, Bowdoin, 1985 (Brunswick, Maine, 1985), 345-414, Proc. Sympos. Pure Math., 46, Part 1, Amer. Math. Soc., Providence, RI, 1987.

Dipartimento di Matematica, Università di Trento, I-38123 Povo (TN)

E-mail address: marco.andreatta@unitn.it, luca.tasin@unitn.it 